\title{
OS EFEITOS DA REABILITAÇÃO VIRTUAL SOBRE O EQUILÍBRIO DE IDOSAS INSTITUCIONALIZADAS
}

\section{THE EFFECTS OF VIRTUAL REHABILITATION ON BALANCE OF INSTITUTIONALIZED ELDERLY}

\author{
Camila Cristina de Castroo ${ }^{a^{*}}$, Camila Silva da Cruz ${ }^{b^{*}}$, Fabiana de Andrade Nascimento ${ }^{c^{*}}$, Jéssica \\ Maiara Trindade Nascimento ${ }^{\mathrm{d}^{*}}$, Elaine Guiomar Baêta ${ }^{\mathrm{e}^{*}}$ \\ camilinha.ccastro@hotmail.comª , camila.csccruz@gmail.com ${ }^{b}$, fabianaandrade.94@hotmail.comc, maiara.jssica@yahoo.com.br ${ }^{\mathrm{d}}$, elainebaeta@ \\ unipac.bre \\ Centro Universitário Presidente Antônio Carlos - UNIPAC - Barbacena/MG*
}

Data de submissão: 27/01/2019 Data de aceite: 18/02/2019

\section{RESUMO}

Introdução: $\mathrm{O}$ envelhecimento causa uma progressiva perda da capacidade de adaptação do indivíduo. Para a reabilitação do equilíbrio, utiliza-se atualmente na fisioterapia, a reabilitação virtual. Objetivo: Avaliar se a nova técnica de reabilitação irá contribuir para a melhora do equilíbrio corporal de idosas institucionalizadas. Métodos: Trata-se de um estudo experimental do tipo intervencional, randomizado de autocontrole. A amostra $(n=14)$ foi distribuída em dois grupos, sendo um grupo experimental, que realizou a Reabilitação Virtual e um grupo controle, que foi submetido apenas à coleta de dados vitais a cada sessão. Os procedimentos da pesquisa foram divididos em três fases. Na $1^{\circ}$ fase foi realizada a avaliação do equilíbrio através da Escala de Equilíbrio de Berg. A segunda fase foi aplicada a reabilitação virtual. Foram realizadas 12 sessões com 02 encontros semanais, tendo duração de 30 minutos à sessão para cada idosa. Ao final, iniciou-se a terceira fase, onde aplicou-se novamente a EEB, sendo feita a análise dos dados obtidos. Resultados: Foram identificados valores da Escala de Equilíbrio de Berg diferentes entre os grupos nos escores pré vs. pós, com valores significativos $(\mathrm{P}<0,05)$ $\mathrm{Na}$ análise individual de cada grupo estudado, não foram encontradas diferenças significativas para os sinais vitais. Conclusão: Este estudo demonstrou que a técnica utilizada mostrou-se de fácil aplicabilidade e eficaz no treinamento de equilíbrio de idosas institucionalizadas, apresentando melhores escores na reavaliação da escala de equilíbrio de Berg.

Palavras-chave: Equilíbrio postural, idoso, reabilitação

\section{ABSTRACT}

Introduction: The aging causes a progressive loss of adaptability of the individual. Nowadays in the Physiotherapy, the virtual rehabilitation is used to the balance rehabilitation. Objective: This study aims to evaluate the new technique of rehabilitation and its contribution to the corporal balance improvement of elderly women. Methods: This is a randomized, selfcontrolled, experimental and an interventional study design. The sample $(n=14)$ was distributed in two groups, and the first group was the experimental one, which realized the rehabilitation, and the second one, the control group, which was subject only to data collection of vital signs in each session. During this research, the procedures were divided in three stages. In stage one was realized an evaluation of balance through the Berg Balance Scale. In the second stage was applied the virtual rehabilitation. At this stage, 12 sessions were realized, each one with 2 weekly meetings, with 30 minutes of length per session and to each elderly woman. In the third stage was again applied the Berg Balance Scale, where was made an analysis of the data collected. Results: It was identified different values on Berg Balance Scale among the groups in the scores pre versus post whit significant values $(\mathrm{P}<0,05)$. In the individual analysis of each studied group there were not found meaningful differences in the vital signs. Conclusion: This study has demonstrated that the used protocol has an easy applicability and it was effective in the balance training for elderly women institutionalized, and it showing the best scores in the reassessment of the Berg Balance Scale.

Keywords: Postural balance, elderly. rehabilitation 


\section{Introdução}

De acordo com o Instituto Brasileiro de Geografia e Estatística (IBGE), o país se encontra em processo de envelhecimento com aumento gradativo de pessoas acima 60 anos em consequência da redução da mortalidade e do aumento da expectativa de vida ${ }^{1}$. Diante desse fato a saúde se torna um importante foco de atenção, pois, o envelhecimento é um processo dinâmico, progressivo e inevitável ${ }^{2}$ que causa perda da capacidade de adaptação do indivíduo reduzindo suas habilidades funcionais para realização das atividades de vida diária (AVD). ${ }^{3,4}$ Nessa perspectiva, a contração muscular mais lenta, o enfraquecimento da constituição óssea, o enrijecimento das articulações, assim como, a diminuição da massa muscular e da condução do estímulo neurale força, sãoalterações morfológicas, funcionais e bioquímicas, que contribuem para o prejuízo no controle postural ${ }^{5}$. O controle postural é resultado da relação entre os sistemas sensoriais (visual, vestibular e somatossensorial), do sistema motor e do sistema nervoso central. ${ }^{6}$ Com o processo de envelhecimento, os sistemas sofrem perdas funcionais que dificultam a execução da resposta motora responsável pela manutenção do controle da postura corporal, isto é, do equilíbrio. Tais alterações tornam os idosos mais vulneráveis a episódios de quedas 5 . A queda é um evento constituído por elementos causais classificados como intrínsecos e extrínsecos. Causas intrínsecas são aquelas relacionadas ao indivíduo, ocasionadas pela instabilidade postural, e as extrínsecas são de caráter externo, como, por exemplo, o tipo de calçado utilizado, o tipo de terreno, a iluminação do ambiente e a acessibilidade aos locais. As consequências das quedas sofridas em idosos são mais graves, pois respondem com mais lentidão e menos eficazmente as alterações ambientais, causando prejuízos funcionais ${ }^{4,5}$ e aumentando os níveis de morbidade e mortalidade nessa população ${ }^{7}$. Os residentes de Instituições de Longa Permanência para Idosos (ILPIs) são considerados uma população complexa devido a fatores associados à morbidade, polifarmácia e a alta prevalência de dependência nas atividades de vida diária. Estima-se que entre 50\% e 66\% dos idosos institucionalizados sofram ao menos uma queda por ano ${ }^{8}$. Na reabilitação do equilíbrio, utiliza-se atualmente na fisioterapia, a reabilitação virtual $(\mathrm{RV})^{9}$, que é uma nova ferramenta terapêutica aplicada em diversas faixas etárias, pois cria um ambiente totalmente virtual, tridimensional de forma lúdica que proporciona aos pacientes estímulos visuais, táteis, auditivos e sensoriais. A RV pode contribuir para a reabilitação da marcha, equilíbrio, postura corporal, coordenação motora, qualidade de vida, dentre outros, além de promover ao paciente interatividade, motivação ao tratamento e melhora do desempenho físico e cognitivo ${ }^{5,10-12}$. Diante do exposto, o objetivo deste estudo é avaliar como a técnica de reabilitação irá contribuir para a melhora do equilíbrio corporal de idosas institucionalizadas.

\section{Materiais e Métodos}

O projeto foi submetido ao Comitê de Ética em Pesquisa (CEP) da Universidade Presidente Antônio Carlos (UNIPAC), sob o parecer de número 2.014.243, com data de aprovação em 12 de abril de 2017, sob registro número CAAE 63739617.4.0000.5156. Para a realização da pesquisa o estudo foi apresentado e autorizado pelos responsáveis de instituições de longa permanência do munícipio de Barbacena/MG.

Utilizou-se uma amostra de conveniência, probabilística devido às características e condições das idosas das instituições envolvidas. Como critérios de inclusão foram utilizados: idosas institucionalizadas, independentes, que têm condição física, intelectual, entre 65 a 85 anos e que aceitaram participar de todas as etapas da pesquisa. Os critérios de exclusão foram: idosas que estavam em tratamento fisioterapêutico na reabilitação vestibular, utilizavam dispositivo de auxílio para deambulação, apresentavam déficits visuais ou auditivos e que se negaram a participar da avaliação e intervenção. De acordo com os critérios adotados a amostra foi composta por 15 idosas, porém no decorrer da pesquisa uma participante veio a óbito, ficando a amostra composta por 14 idosas.

Todos os procedimentos realizados foram previamente explicados as idosas convidadas a participarem da pesquisa. As que estavam de 
acordo em participar do estudo voluntariamente assinaram o Termo de Consentimento Livre e Esclarecido (TCLE). Realizou-se um sorteio para a distribuição dos grupos, sendo um grupo experimental (GE), que realizou a Terapia Virtual e um grupo controle (GC), que foi submetido à coleta de dados vitais a cada sessão. Os procedimentos da pesquisa foram divididos em três fases. Na primeira fase foi realizada a avaliação do equilíbrio através da Escala de Equilíbrio de Berg (EEB), que é um instrumento validado, de alta confiabilidade para a avaliação do equilíbrio funcional em idosos. A escala avalia o indivíduo em 14 situações de atividades do dia a dia, sentado para em pé, em pé sem apoio, sentado sem apoio, em pé para sentado, transferências, em pé com olhos fechados, em pé com os pés juntos, reclinar à frente com os braços estendidos, apanhar objeto do chão, virando-se para olhar para trás, girando 360 graus, colocar os pés alternadamente sobe um banco, em pé com um pé em frente ao outro e em pé apoiando em um dos pés. Cada uma das situações foi pontuada numa escala de 0 a 4 pontos, com a pontuação máxima de 56 pontos ${ }^{12,14}$. Durante a segunda fase foi aplicada a reabilitação virtual, onde foram utilizados os seguintes materiais: Vídeo Game X-box360 Microsoft $^{\oplus}$, Kinect que capta a movimentação dos participantes por meio de um dispositivo em forma de sensor, sem a necessidade do uso 9 do controle, possibilitando, dessa forma, maior liberdade de movimento ao jogador. $\mathrm{O}$ Kinect foi posicionado há uma altura de 1,6 metros do chão. O televisor utilizado foi da marca LG, modelo 32LS3400 DTV, resolução 1366x768, ângulo de visão $178^{\circ} \times 178^{\circ}, 32^{\prime}$ polegadas, ele foi colocado sobre uma mesa de 1 metro de altura. O game utilizado para intervenção foi o Kinect Adventures Corredeiras. Nessa fase foi realizada a familiarização das voluntárias com o jogo, que simula estar dentro de um bote descendo por um rio turbulento até chegar a um local seguro. No caminho é preciso coletar itens para conseguir pontos, com isso é exigido a participante pequenos saltos, realizar inclinações para a direita e para a esquerda, manter os braços abduzidos e a base levemente alargada. O jogo possui três fases, sendo elas: Riacho sinuoso (nível difícil), Cavernas aconchegantes (nível intermediário) e Riacho forte (nível básico). As fases se alteravam de acordo com a evolução da participante. Durante o jogo, a participante se posicionava individualmente a uma distância de aproximadamente 2 metros da frente do televisor para que o sensor pudesse captar seus movimentos. O local para a realização da atividade era amplo, coberto com piso e sem objetos ao redor. As participantes utilizaram calçados fechados que não soltavam de seus pés durante a realização das atividades, garantindo assim sua segurança. Foram realizadas 12 sessões com 02 encontros semanais, cada sessão tendo duração de 30 minutos para cada idosa, sendo iniciada e finalizada com aferição da PA, FC, FR e SpO2 para controle e obtenção de dados vitais, antes e após atividade. Todas as idosas foram devidamente assistidas e auxiliadas durante a realização dos exercícios para evitar os possíveis riscos de quedas. Para a coleta dos dados no decorrer da pesquisa foram utilizados: 02 esfigmomanômetros aneróide Premium ${ }^{\oplus}$, registro ANVISA/MS 10385180030 composto por 01 manômetro $(0-300 \mathrm{mmHg}), 01$ braçadeira, 01 válvula de deflação e 01 pêra, 02 1estetoscópios Rappaport Premium ${ }^{\circledR}$, registro ANVISA/MS 80275310014 composto por 01 par de olivas rosqueável, 02 membranas de plástico rígido, anel rosqueado de cobre, câmara de som em liga de alumínio, tubo rotatório central, tubo duplo de PVC, fone biauricular, diafragma aberto e auscultador para a aferição da PA; 02 oxímetros de pulso ChoiceMMed ${ }^{\circledast}$, registro ANVISA $\mathrm{n}^{\circ}$ 10349590060 do modelo MD300C1 para controle da FC e SpO2; 02 relógios de pulso para conferência da FR. Ao final das 12 sessões, foi iniciada a terceira fase, onde aplicou-se novamente a EEB, sendo feita a análise dos dados obtidos e verificando se houve melhora do equilíbrio após a intervenção. A média da pontuação obtida na escala foi o meio quantitativo para obtenção e comparação dos dados pré vs. pós da terapia virtual.

A estatística utilizada foi através de análise descritiva e utilizaram-se médias \pm desvio padrão para os dados obtidos pré vs. pós para efeito treinamento na pontuação da Escala de Berg. Os valores da primeira sessão dos sinais vitais de Pressão Arterial, Frequência Cardíaca, Frequência Respiratória e Saturação de Oxigênio foram testados no dia um, para avaliar o comportamento agudo após uma sessão da reabilitação virtual em idosas. A normalidade dos dados foi testada pelo 
teste de Shapiro-Wilk, o Teste T-Student (amostras independentes) para as variáveis com distribuição normal e o Teste U-Mann Witney para as variáveis com distribuição não normal. A Análise de Variância - ANOVA, de duas entradas com medidas repetidas foi utilizada para identificar o comportamento durante a realização o período da reabilitação virtual com a utilização da Escala de Berg. Todas as análises foram realizadas no software SPSS 24.0 Windows ${ }^{\circledast}$ (IBM Corporation, New York, EUA) e o nível de significância adotado foi de $\alpha=0,05$.

\section{Resultados}

A amostra contou com 14 participantes, sendo sete do grupo experimental (50\%) e sete do grupo controle (50\%), as quais foram avaliadas através da escala de equilíbrio de Berg. A média das idades foi de 75 anos, sendo que a maioria $(73,3 \%)$ encontrava-se entre 70 e 79 anos. Para os sinais vitais, do primeiro dia de intervenção entre os Grupos Experimental (GE) e Controle (GC), os valores pré vs. pós reabilitação virtual como $\mathrm{PAS}, \mathrm{PAD}, \mathrm{FC}$, FR e SpO2, foram comparados pela média \pm desvio padrão, após testados os pressupostos de normalidade, os dados estão na tabela 01. Em análise individual a cada grupo estudado, verificou-se que não foram encontradas diferenças significativas para os sinais vitais. Tabela 01. Comparação dos Sinais Vitais PAS, PAD, FC, FR e SpO2 dos GE e GC antes e após uma sessão de Reabilitação Virtual $(\mathrm{P}>0,05)$

Tabela 1 - Comparação dos Sinais Vitais PAS, PAD, FC, FR e SpO dos GE e GC Antes e Após uma Sessão de Reabilitação Virtual $(\mathrm{P}>0,05)$.

\begin{tabular}{cccc}
\hline N=14 & PAS pré & PAS pós & p valor \\
\hline$(\mathrm{GE})$ & $128,57 \pm 10,6$ & $131,43 \pm 6,9$ & 0,675 \\
$(\mathrm{GC})$ & $125,71 \pm 13,9$ & $127,14 \pm 11,1$ & \\
\hline$(\mathrm{GE})$ & PAD pré & PAD pós & 0,403 \\
$(\mathrm{GC})$ & $72,86 \pm 4,8$ & $87,14 \pm 4,8$ & \\
\hline$(\mathrm{GE})$ & $75,71 \pm 7,8$ & $84,29 \pm 11,3$ & 0,430 \\
$(\mathrm{GC})$ & $\mathbf{F C}$ pré & FC pós & \\
\hline & $89,71 \pm 16,8$ & $96,14 \pm 13,5$ & 0,552 \\
\hline$(\mathrm{GE})$ & $82,29 \pm 9,8$ & $81,71 \pm 8,3$ & \\
\hline$(\mathrm{GC})$ & $\mathbf{F R}$ pré & $\mathbf{F R ~ p o ́ s ~}$ & 0,06 \\
\hline & $15,43 \pm 1,1$ & $18,00 \pm 1,5$ & \\
\hline$(\mathrm{GE})$ & $15,43 \pm 1,1$ & $15,43 \pm 1,1$ & $\mathbf{S a O}$ pós \\
\hline
\end{tabular}

Legenda: $\mathrm{GE}=$ Grupo Experimental. GC $=$ Grupo Controle. $\mathrm{FC}=$ Frequência Cardíaca. $\mathrm{FR}=$ Frequência Respiratória. PAS e PAD = Pressão Arterial Sistólica e Pressão Arterial Diastólica. SpO2 = Sauração de Oxigênio

A ANOVA de dois caminhos com medidas repetidas, e o Post Hoc de Bonferroni foram usados para identificar as diferenças Pré vs. Pós entre os GE $(48,00 \pm 2,7$ vs. $54,00 \pm 1,15$ pontos $)$ e GC $(43,00 \pm 8,8$ vs. $46,86 \pm 9,5$ pontos), durante o Treinamento com a Reabilitação Virtual para as pontuações obtidas na Escala de Equilíbrio de Berg. Foram identificados valores da Escala diferentes entre o GE e GC e nos escores pré vs. pós, em ambos com efeito treinamento significativos $(\mathrm{P}<0,05)$. Os dados estão demonstrados no gráfico. 
Gráfico 1 - Pontuação Pré vs. Pós a Reabilitação Virtual no Grupo Controle e Experimental em Idosas.

Pontuação Pré vs. Pós de Berg Antes e Após a Reabilitação Virtual em Idosas

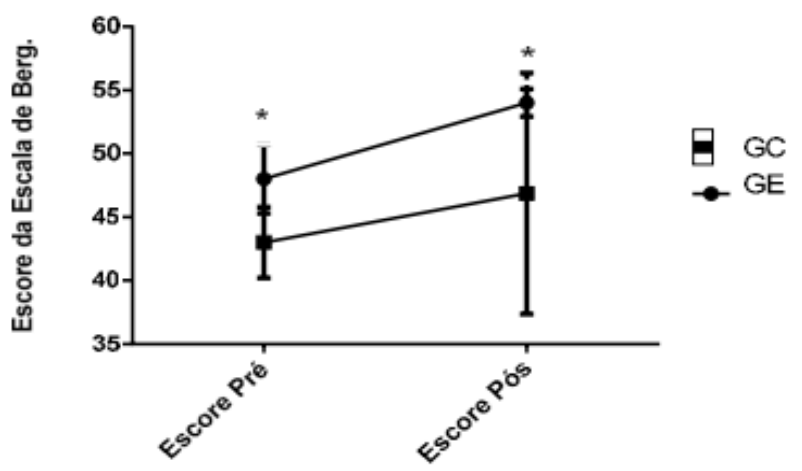

Fonte: Autor

\section{Discussão}

A população idosa institucionalizada apresenta perfildeisolamento social, inatividadeefragilidade, o que pressupõe o declínio de capacidades físicas, além da deterioração das capacidades fisiológicas naturais inerentes ao próprio processo de envelhecimento $^{7}$. O envelhecimento tem fatores intrínsecos sendo eles, perda de equilíbrio, alterações na massa muscular e óssea e debilidade do controle postural ${ }^{15}$. A realidade virtual é uma tecnologia que permite ao terapeuta uma grande variedade de estímulos com maior especificidade se comparada a métodos tradicionais de reabilitação, apresentando ao paciente conflitos sensoriais em diferentes níveis de dificuldades e em ambiente segur ${ }^{16}$. No presente estudo, não foram encontradas diferenças significativas em relação aos sinais vitais coletados antes e após a intervenção com a RV. Adverso a estes achados a resposta pressórica e a $\mathrm{SpO} 2$ pós exercício, Borges e Mendes ${ }^{17}$ demonstraram em seus estudos que houve diferença na resposta dos indivíduos, antes e após a realização do exercício físico, e que em $83,3 \%$ dos idosos participantes houve uma diminuição da PAS e PAD. Pode- se observar, também, na maioria dos idosos, após o treino com a Xbox terapia, que a $\mathrm{PA}$ e a $\mathrm{SpO} 2$ tiveram uma diminuição do valor em relação ao início do atendimento. Já em relação a FR observou-se um aumento em 5 indivíduos, os resultados em relação a FC foram similares aos encontrados neste estudo, em que a mesma manteve valores semelhantes antes e após o exercício. Os resultados deste trabalho mostram significância após as 12 sessões de Reabilitação Virtual com o uso do Xbox 360 e kinect, apresentando melhores escores na reavaliação da Escala de Equilíbrio de Berg, tanto para o GE e GC. Imagética (IM), é a repetição de movimentos a partir da imaginação dos mesmos, sem a execução motora efetiva. Através desta, são ativados circuitos neurais semelhantes aos que são envolvidos quando o indivíduo efetua um dado ato motor, ativando as redes responsáveis pelo planejamento e controle motor ${ }^{18}$. As participantes do grupo controle estavam presentes durante a aplicação da RV, devido a isso, há uma possibilidade de ter ocorrido à imagética motora nessas participantes, explicando o resultado do aumento do score neste grupo, que mesmo não participando da intervenção obteve melhora no equilíbrio. Cardoso et al. ${ }^{19}$ avaliando o efeito da imagética no equilíbrio durante a marcha de pacientes com doença de Parkinson, obtiveram resultados positivos, mostrando que a imagética se encontra associada positivamente com melhora da marcha e equilíbrio. Os dados mostram que, assim como no GE o estudo de Barros et al. ${ }^{20}$, verificou aumento significante nos scores médios do teste de equilíbrio de Berg, mostrando que, em um curto período de treinamento de realidade virtual (nove sessões - três semanas), o risco de queda foi reduzido. Os resultados são comparáveis com a pesquisa de Jaime et al. ${ }^{21}$ e Rendon et al. ${ }^{22}$ que avaliou o uso da reabilitação virtual para melhora do equilíbrio dinâmico em idosos. Jaime et al. ${ }^{21}$ teve como objetivo verificar se o uso de jogos que precisam do acessório Kinect tinha a capacidade de melhorar o equilíbrio em idosos institucionalizados. Ambos apresentaram um aumento significantemente na reavaliação do equilíbrio. Comparando os efeitos da RV com métodos convencionais para melhora do equilíbrio, Park et al. ${ }^{23}$ selecionaram 30 idosos com mais de 65 anos, que realizaram exercícios durante 30 minutos, 3 vezes por semana, durante 8 semanas. Os resultados revelam que houve diferenças significativas entre antes e após o experimento com o grupo que realizou a terapia virtual, enquanto que 
nenhuma diferença significativa foi encontrada no grupo com exercícios convencionais. Penassol et al. ${ }^{24}$ comparando os efeitos da RV com a cinesioterapia, para melhora da percepção visual, mobilidade funcional, ajuste postural e ganho de equilíbrio em idosos, observaram que ambas as intervenções trouxeram resultados satisfatórios a amostra estudada. Então, trazendo-se a luz o que foi observado por Tsang e $\mathrm{Fu}^{25}$ com 79 idosas institucionalizados, divididos em dois grupos após 6 semanas de treinamento, observou-se que o grupo RV alcançou um maior desempenho na melhora do equilíbrio comparando ao grupo que realizou treinamento de equilíbrio convencional. São escassos na literatura brasileira, estudos que utilizaram o X-box 360 como ferramenta de reabilitação para melhora do equilíbrio de idosos institucionalizados. Portanto, este estudo se torna importante, pois de acordo com os resultados encontrados, pode iniciar uma nova modalidade de tratamento. A presente pesquisa teve algumas limitações, tais como: determinação da faixa etária, literatura acerca do tema e disponibilidade das participantes (amostra).

\section{Conclusão}

Os resultados obtidos com a realização do presente estudo demonstraram que a técnica utilizada se mostrou de fácil aplicabilidade e eficaz no treinamento de equilíbrio de idosas institucionalizadas, apresentando melhores escores na reavaliação da escala de equilíbrio de Berg, além de oferecer motivação durante o tratamento se comparado as outras formas de reabilitações convencionais. Há carência de estudos que empregam a reabilitação virtual no equilíbrio de idosas institucionalizadas, demonstrando a necessidade da realização de novas pesquisas sobre o tema, com a utilização de uma amostra maior, sendo possível verificar a aplicabilidade desse protocolo em idosos, objetivando o ganho de equilíbrio favorecendo o método baseado em evidências.

\section{Referências}

1. Rebelatto JR, Castro APD, Sako FK, Aurichio TR. Equilíbrio estático e dinâmico em indivíduos senescentes e o índice de massa corporal. FisioterMov. 2008; 21(3):6975 .

2. Barcala L, Colella F, Araujo MC, Salgado ASI, Oliveira CS. Análise do equilíbrio em pacientes hemiparéticos após o treino com o programa Wii Fit. Fisioter. mov. 2011; 24(2):337- 43.

3. Silva AFG, Berbel AM. O benefício da dança sênior em relação ao equilíbrio e às atividades de vida diárias no idoso. ABCS healthsci. 2015; 40(1).

4. Albino ILR, Freitas CDLR, Teixeira AR, Gonçalves AK, Santo AMPVD, Bós ÂJG. Influência do treinamento de força muscular e de flexibilidade articular sobre o equilíbrio corporal em idosas. Revista brasileira de geriatria e gerontologia. RJ. 2012; Vol. 15, n. 1: p. 17- 25.

5. Silva RR, Iwabe-Marchese C. Uso da realidade virtual na reabilitação motora de uma criança com Paralisia Cerebral Atáxica: estudo de caso. Fisioterapia e Pesquisa. 2015; 22(1): 97-102.

6. Maciel ACC, Guerra RO. Prevalência e fatores associados ao defícit de equilíbrio em idosos. R. bras. Ci e Mov. 2005; 13(1):37-44. 7. Santos FPV, Borges LL, Menezes RL. Correlação entre três instrumentos de avaliação para risco de quedas em idosos. Fisiot.Movimento. 2013; 26(4)

7. Moreira, LA. Efeitos da reabilitação virtual no equilíbrio e mobilidade em idosos institucionalizados. Rio Claro, 2017.

8. Treml CJ, Kalil FFA, Ciccarino RFL, Wegner R, Saita CYDS, Corrêa AG. O uso da plataforma Balance Board como recurso fisioterápico em idosos. Rev. bras. Geriatr. Gerontol. 2013; 16(4):759-768.

9. Mendes FADS, Arduini L, Botelho A, Cruz MBD, Santos CPCC, Pompeu SMAA, Pompeu JE. Pacientes com a Doença de Parkinson são capazes de melhorar seu desempenho em tarefas virtuais do Xbox Kinect ${ }^{\circledR}$ : uma série de casos. Motricidade. 2015; 11(3): 68-80

10. Batista PKA. Aplicação de protocolo de equilíbrio em jovens com sintomas de disfunções vestibulares: um estudo de caso. Univers. Estad.da Paraíba, Campina Grande. 2013.

11. Schiavinato AM, Machado BDC, Pires MDA, Baldan C. Influência da realidade virtual no equilíbrio de paciente portador de disfunção cerebelar- Estudo de caso. RevNeurocienc. 2011; 19(1):119-127.

12. Miyamoto ST et al. Brazilian version of the Berg balance scale. Braz J Med Biol Res [online]. 2004; vol.37, n.9: pp.1411-1421. 
13. Batista JS, Wibelinger LM, Marchi, ACB, Pasqualotti A. Evaluation and physiotherapeutic intervention in older with deficit balance through the Scale of Berg and Wii Balance Board platform. Fisioterapia em Movimento. 2014; 27(1): 21-28.

14. Marques AKMC, Moraes KRF, Melo AM, Alves JSM. Efeitos da Reabilitação Virtual no treinamento de equilíbrio de idosos. Anais CIEH. 2015; vol.2: N.1.

15. Garcia AP, Ganança MM, Cusin FS, Tomaz A, Ganança FF, Caovilla HH. Vestibular rehabilitation with virtual reality in Ménière's disease. Brazilian journal of otorhinolaryngology. 2013, 79(3): 366-374.

16. Borges APM, Mendes GC. Avaliação cognitiva e de equilíbrio em idosos institucionalizados após intervenção de Xbox terapia. Saúde. 2015; 1(13).

17. Santiago LMDM. Efeitos da prática mental na marcha de indivíduos com doença de parkinson: ensaio clínico randomizado. (Master's thesis, Universidade Federal do Rio Grande do Norte); 2013.

18. Cardoso AG, Sasaki AC, Mendel T. Efeitos da Imagética no Equilíbrio Durante a Marcha de Pacientes com Doença de Parkinson. Anais da MCC, Salvador, 2012; 1(3).

19. Barros GWP, Silva LRG, Santos WR, Filho HT, Santos WR. Impacto do tratamento com realidade virtual no risco de quedas em idosos. Revista da Universidade Vale do Rio Verde. 2016; 14(1): 279-285.

20. Jaime E, Cardoso T, Nogueira M, Mendonça R, Alves F, Alves A, et al. A influência do Kinect para melhora do equilíbrio, força e agilidade em idosos institucionalizados da cidade de São Luis de Montes Belos - GO. Revista Eletrônica Faculdade Montes Belos. 2016; 9(1).

21. Rendon AA, Lohman EB, Thorpe D, Johnson EG, Medina E, Bradley B. The effect of virtual reality gaming on dynamic balance in older adults. Age and ageing. 2012; 41(4): 549- 552.

22. Park EC, Kim SG, Lee CW. The effects of virtual reality game exercise on balance and gait of the elderly. Journal of physical therapy science.2015; 27(4): 1157-1159.

23. Panassol FP, Oltramari G, Schuster RC. Efeitos da realidade virtual no equilíbrio de idosos saudáveis. Revista interdisciplinar ciências médicas-MG. 2017; 1(1): 79-95.
24. Tsang WW, Fu AS. Virtual reality exercise to improve balance control in older adults at risk of falling. Hong Kong medical journal. 2016; 22: 19-22

\section{Como citar este artigo:}

Castro CC, Cruz CS, Nascimento FA, Nascimento JMT, Baêta EG. Efeitos da reabilitação virtual no equilíbrio de idosas institucionalizadas. Rev. Aten. Saúde. 2019;17(60): 47-53 